\title{
Relative validation of fruit and vegetable intake and fat intake among overweight and obese African-American women
}

\author{
Iris Alcantara ', Regine Haardörfer ${ }^{1,2}$, Julie A Gazmararian ${ }^{1,3}$, Terry J Hartman ${ }^{3}$, \\ Brenda Greene ${ }^{4}$ and Michelle C Kegler ${ }^{1,2, *}$ \\ ${ }^{1}$ Emory Prevention Research Center, Department of Behavioral Sciences and Health Education, Rollins School of \\ Public Health, Emory University, 1518 Clifton Road NE, Atlanta, GA 30322, USA: ${ }^{2}$ Department of Behavioral \\ Sciences and Health Education, Rollins School of Public Health, Emory University, Atlanta, GA, USA: ${ }^{3}$ Department of \\ Epidemiology, Rollins School of Public Health, Emory University, Atlanta, GA, USA: ${ }^{4}$ Southwest Health District, \\ Georgia Department of Public Health, Albany, GA, USA
}

Submitted 2 August 2013: Final revision received 31 July 2014: Accepted 1 September 2014: First published online 21 November 2014

\begin{abstract}
Objective: To compare commonly used dietary screeners for fat intake and fruit and vegetable intake with $24 \mathrm{~h}$ dietary recalls among low-income, overweight and obese African-American women.

Design: Three telephone interviews were completed; measures included two $24 \mathrm{~h}$ dietary recalls (a weekday and weekend day) using the Nutrition Data System for Research software, the Behavioral Risk Factor Surveillance System's (BRFSS) Fruit and Vegetable Consumption Module and the National Cancer Institute's (NCI) Percentage Energy from Fat Screener.

Setting: Participants were recruited from three federally qualified health centres in south-west Georgia, USA.

Subjects: Participants ( $n$ 260) were African-American women ranging in age from 35 to 65 years. About half were unemployed (49.6\%) and 58.7\% had a highschool education or less. Most were obese (88.5\%), with 39.6\% reporting a BMI $\geq 40 \cdot 0 \mathrm{~kg} / \mathrm{m}^{2}$.

Results: Mean fruit and vegetable intake reported from the $24 \mathrm{~h}$ dietary recall was 2.66 servings/d compared with 2.79 servings/d with the BRFSS measure. The deattenuated Pearson correlation was $0 \cdot 22$, with notable variation by weight status, education level and age. Mean percentage of energy from fat was $35.5 \%$ as reported from the $24 \mathrm{~h}$ dietary recall, compared with $33.0 \%$ as measured by the NCI fat screener. The deattenuated Pearson correlation was $0 \cdot 38$, also with notable variation by weight status, education level and age.

Conclusions: Validity of brief dietary intake measures may vary by demographic characteristics of the sample. Additional measurement work may be needed to accurately measure dietary intake in obese African-American women.
\end{abstract}

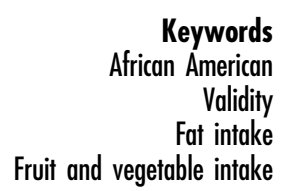

Keywords

Validity

at intake

Fruit and vegetable intake
Significant racial and ethnic disparities in overweight and obesity prevalence exist, with higher age-adjusted prevalence among non-Hispanic blacks (76.7\%) and Hispanics $(78.8 \%)$ compared with non-Hispanic whites $(66.7 \%)^{(1)}$. Non-Hispanic black women aged 40 years or older are particularly affected by the overweight and obesity epidemic, with $87.7 \%$ of $40-59$-year-olds being overweight or obese compared with $61.3 \%$ of nonHispanic whites and $86.4 \%$ of those aged 60 years and over being overweight or obese compared with $71.6 \%$ of non-Hispanic whites ${ }^{(1)}$. Additionally, non-Hispanic black women have a higher combined prevalence of class II $\left(B M I \geq 35 \cdot 0 \mathrm{~kg} / \mathrm{m}^{2}\right.$ ) and class III obesity (BMI $\geq 40 \cdot 0 \mathrm{~kg} / \mathrm{m}^{2}$ ) compared with non-Hispanic whites, with $26 \cdot 0 \%$ classified as class II and III obese compared with $14.4 \%$, respectively ${ }^{(1)}$. Similarly, geographic disparity exists in weight status, with national estimates for obesity being higher in rural regions of the USA compared with urban areas ${ }^{(2,3)}$. Data from the 2005-2008 National Health and Nutrition Examination Survey indicated that the obesity prevalence in rural areas was $39.6 \%$ compared with $33.4 \%$ in urban areas ${ }^{(3)}$.

Dietary intake remains an important focus in the prevention of overweight and obesity. Consumption of less energy and fatty foods, and more fruits and vegetables, is associated with lower $\mathrm{BMI}^{(4)}$. Consequently, total energy, fat and fruit and vegetable intakes are commonly measured to 
study dietary intake and its relationship with chronic disease, weight gain prevention and weight loss. Multiple $24 \mathrm{~h}$ dietary recalls are often used to measure dietary intake and frequently used to validate other self-reported dietary measures such as FFQ and targeted screening instruments ${ }^{(5-7)}$.

Shorter dietary measures such as screeners have been developed and validated against $24 \mathrm{~h}$ dietary recalls to address feasibility limitations posed by collecting multiple $24 \mathrm{~h}$ food records ${ }^{(8)}$. Such instruments include the Fruit and Vegetable Consumption Module (hereafter, 'FV module') from the Behavioral Risk Factor Surveillance System (BRFSS) ${ }^{(9)}$ and the Percentage Energy from Fat Screener (hereafter, 'fat screener') developed by the National Cancer Institute $(\mathrm{NCI})^{(10,11)}$. Both instruments have been widely used to measure fruit and vegetable intake, and fat intake, respectively, and have been shown to be acceptable in terms of both reliability and validity $^{(9-15)}$. One study evaluating the use of the BRFSS FV module in a diverse population (diverse in age, gender, education and geographic location) found that the mean daily consumption of fruits and vegetables measured by the module was comparable to the mean daily consumption measured by food records or recalls ${ }^{(9)}$. Data from that study were collected from participants living in different states and although the correlation coefficients between the BRFSS FV module and the food records or recalls varied across subgroups, they were moderate in agreement and ranged from 0.34 to $0.66^{(9)}$. Similarly, another study evaluated the use of the NCI fat screener and found moderate correlations between true intakes and the screener, ranging from 0.58 for women and 0.64 for men ${ }^{(10)}$. Mean percentage energy from fat was also comparable, with an estimated $30 \cdot 1 \%$ from two $24 \mathrm{~h}$ dietary recalls and $29.9 \%$ from the NCI fat screener ${ }^{(10)}$.

To our knowledge, the validity of these instruments has not been extensively explored among high-priority sub-populations, in particular among low-income AfricanAmerican women. Factors such as race/ethnicity, BMI, education level and age may impact validity ${ }^{(7,16-18)}$. The NCI fat screener, for example, did not perform as well in a study site comprised mostly of African-American participants compared with other sites with fewer AfricanAmerican study participants ${ }^{(11)}$. These investigators, much like others, suggested that dietary instruments used in studies be tailored to measure the specific diet of the target population $^{(7,11)}$. Additionally, Marks et al. have found that BMI, occupation, age, gender, education and the presence of any medical condition are significantly associated with differences in the validity of measures of certain nutrient intakes and/or energy intake ${ }^{(16,17)}$. Educational attainment and literacy can also affect validity ${ }^{(18)}$. These factors may moderate validity in a number of ways: BMI may impact social desirability and self-reported dietary intake (underreporting or over-reporting); age may impact recall; and literacy may impact the ability of the respondent to understand and interpret dietary instruments.
Characterizing the associations between the BRFSS FV module, the NCI fat screener and repeated, unannounced, telephone $24 \mathrm{~h}$ dietary recalls within an overweight and obese African-American population will contribute to a long-term goal of understanding the performance of these instruments in a variety of settings. We expected that selfreported fruit and vegetable intake as measured by the BRFSS FV questions would at best moderately correlate with fruit and vegetable intake assessed by $24 \mathrm{~h}$ recalls. We expected the NCI fat screener to perform likewise with respect to dietary fat intake. Further, we hypothesized that the two instruments would perform similarly when examining correlations across categories of BMI, education, employment and age, with self-reported fruit and vegetable intake showing stronger correlations among African-American women who are overweight (compared with obese), college educated (compared with those with less educational attainment), younger and employed.

\section{Methods}

Data presented here were collected as part of a larger study called Healthy Homes/Healthy Families, a randomized controlled trial testing the effectiveness of a homebased coaching intervention to promote healthier home food and physical activity environments, with the goal of preventing weight gain over time. Participants were recruited in partnership with three federally qualified health centres, spanning nine clinic sites. Participants were females aged $35-65$ years with $\mathrm{BMI} \geq 25 \cdot 0$ years, who lived with at least one other person and resided within 30 miles $(\sim 48 \mathrm{~km})$ of the referring clinic.

Trained interview staff collected data at baseline and at 6 and 12 months post-baseline via telephone interviews. At each data collection point, participants were asked to complete telephone interviews that included two $24 \mathrm{~h}$ dietary recalls (one weekday and one weekend day), the BRFSS FV module and the NCI fat screener. Baseline data collection took place over an average of $34.4 \mathrm{~d}$; the average time elapsed between the two recalls was $12 \cdot 8 \mathrm{~d}$, with over $70 \%$ of recalls being completed within two weeks. The screeners were administered during the third interview right after the second dietary recall. The study was approved by the Emory University Institutional Review Board and verbal informed consent was obtained by telephone. Dietary intake data presented here are from baseline only, prior to any intervention-related encounters, and include only African-American participants.

\section{Measures}

All instruments were self-reported and administered by trained telephone interviewers. Dietary intake data from the BRFSS FV module, the NCI fat screener and two $24 \mathrm{~h}$ dietary recalls were included in analyses, along with BMI, age, employment and education level. 


\section{Fruit and vegetable intake}

Six items from the 2005 BRFSS FV module were used to assess fruit and vegetable intake ${ }^{(19)}$. Items were assessed in frequencies of per day, week, month or year. Total intake per day was calculated as a sum from the individual items.

\section{Percentage of daily energy from fat}

The NCI fat screener included seventeen items that assessed the percentage of daily energy from fat ${ }^{(10,11)}$. Participants were asked about the frequency with which they ate or drank a range of items in the past 12 months. Response categories were as follows: 'never', 'less than once per month', '1-3/month', '1-2/week', '3-4/week', '5-6/week', '1/day' and '2+/day'. Percentage energy from the fat screener was determined using the 2003 scoring procedures from NCI (http://riskfactor.cancer.gov/diet/ screeners/fat/scoring.html).

\section{Twenty-four-bour dietary recall}

Dietary intake, including fruit and vegetable intake and fat intake, was collected and analysed using the Nutrition Data System for Research (NDSR) software version 2010, developed by the Nutrition Coordinating Center, University of Minnesota, Minneapolis, MN, USA ${ }^{(20-22)}$. Two recalls were collected per participant, with one weekday and one weekend recall, administered at two separate interviews. Participants were mailed a copy of a Foods Amount Booklet to assist with estimating portion sizes reported during the recall interviews. The first recall was followed by a physical activity measure; the second recall preceded the BRFSS FV module and the NCI fat screener. Fruit, fat and vegetable intakes can be assessed using NDSR, along with intakes of energy and other nutrients and food groups. Fruit and vegetable intake was determined by summing all individual fruit and vegetable servings from the NDSR output (except for any intake of fried potatoes) for each of the two recalls. Fat intake was used as provided in the NDSR output.

\section{Demographics}

Age (date of birth), race/ethnicity, employment status, annual household income, marital status and education level were assessed using items adapted from the 2005 BRFSS survey ${ }^{(19)}$.

\section{$B M I$}

Self-reported weight (without shoes, in pounds) and height (in feet and inches) were assessed at baseline using two items from the 2005 BRFSS survey ${ }^{(19)}$ and used to calculate BMI. BMI $<18.5 \mathrm{~kg} / \mathrm{m}^{2}$ is considered underweight, $18.5-24.9 \mathrm{~kg} / \mathrm{m}^{2}$ is healthy weight, $25.0-29.9 \mathrm{~kg} / \mathrm{m}^{2}$ is overweight and BMI $\geq 30.0 \mathrm{~kg} / \mathrm{m}^{2}$ is considered obese, with class I, class II and class III obesity defined as BMI of $30 \cdot 0-34.9 \mathrm{~kg} / \mathrm{m}^{2}, 35 \cdot 0-39.9 \mathrm{~kg} / \mathrm{m}^{2}$ and $\geq 40 \cdot 0 \mathrm{~kg} / \mathrm{m}^{2}$, respectively.

\section{Neighbourbood type}

Participants were asked to describe their neighbourhoods as 'in town', 'in the country or rural area with neighbours close by' or as 'in the country with very few neighbours close by'. The question and response options were created by the study team using qualitative data from a previous study $^{(23)}$.

\section{Statistical analyses and power}

Power calculations were based on detecting a significant intervention effect for the larger intervention trial; thus sample size was not determined with the stratified analyses conducted for the present paper in mind. The analyses included all participants with complete data for the three instruments. No exclusion of very high or very low energy intakes was made. The intake of fruits and vegetables as well as the percentage of energy from fat were calculated as means from the two $24 \mathrm{~h}$ dietary recalls. Descriptive statistics for data collected with all three instruments were calculated. Deattenuated Pearson correlation coefficients for fruit and vegetable intake (BRFSS FV module and $24 \mathrm{~h}$ dietary recall data) and fat intake (NCI fat screener and $24 \mathrm{~h}$ dietary recall data) were calculated accounting for within-person variation from the dietary recalls ${ }^{(24)}$. Bland-Altman plots were used to examine agreement between the variables estimated by the $24 \mathrm{~h}$ recall and the shorter instruments across a range of intakes. This technique plots the difference between the methods against the mean of the two methods for each participant ${ }^{(25)}$. Differences were calculated by subtracting the NDSR fruit and vegetable intake value from the BRFSS intake value, and the NDSR value for percentage of energy from fat from the NCI fat screener value. Analyses were performed with and without outliers, with outliers retained in the analyses reported here. All analyses were conducted using the statistical software package SAS version $9 \cdot 3$.

\section{Results}

Over a 22-month recruitment period (February 2011December 2012), a total of 945 women were referred to the study. Of the 945 women, 510 (54\%) were enrolled in the study; 197 were non-responders to call attempts and letters (21\%), 161 refused to participate (17\%), sixty-four were ineligible $(7 \%)$ and thirteen were dropped from recruitment for various reasons (1\%). Of those enrolled, 260 African-American women completed all dietary instruments including both baseline $24 \mathrm{~h}$ dietary recalls, the BRFSS FV module and the NCI fat screener.

The participants in the sample were mostly unemployed or retired (55.00\%) and low income $(71.15 \%$ reported an annual household income below \$US 25000; Table 1). Most were single $(52.35 \%)$ and had high-school education or less $(58.69 \%)$. A majority lived in town, but about $45 \%$ of participants reported residing in a rural area. 
Table 1 Description of the study participants: African-American women ( $n$ 260) aged 35-65 years, south-west Georgia, USA, February 2011-December 2012

\begin{tabular}{|c|c|c|}
\hline & $n$ & $\%$ \\
\hline \multicolumn{3}{|l|}{ Age (years) } \\
\hline $35-44$ & 80 & $30 \cdot 77$ \\
\hline 45-54 & 101 & 38.85 \\
\hline $55-65$ & 79 & $30 \cdot 38$ \\
\hline \multicolumn{3}{|l|}{ Employment } \\
\hline Unemployed & 129 & 49.62 \\
\hline Retired & 14 & 5.38 \\
\hline Part time & 26 & $10 \cdot 00$ \\
\hline Full time & 91 & 35.00 \\
\hline \multicolumn{3}{|l|}{ Annual income } \\
\hline \$US 10000 and under & 91 & 35.00 \\
\hline \$US $10001-25000$ & 94 & $36 \cdot 15$ \\
\hline \$US $25001-50000$ & 54 & $20 \cdot 77$ \\
\hline More than \$US 50000 & 16 & $6 \cdot 15$ \\
\hline \multicolumn{3}{|l|}{ Marital status } \\
\hline Married/living with partner & 110 & 47.65 \\
\hline Single & 150 & $52 \cdot 35$ \\
\hline \multicolumn{3}{|l|}{ Education } \\
\hline Less than high-school diploma or GED & 56 & $21 \cdot 62$ \\
\hline High school or GED & 96 & 37.07 \\
\hline Some college/technical school & 76 & $29 \cdot 34$ \\
\hline College graduate & 31 & 11.97 \\
\hline \multicolumn{3}{|l|}{ Neighbourhood type } \\
\hline In town & 142 & $54 \cdot 62$ \\
\hline In the country or rural area with neighbours close by & 101 & 38.85 \\
\hline In the country with very few neighbours close by & 17 & 6.54 \\
\hline \multicolumn{3}{|l|}{ Weight status } \\
\hline Overweight & 30 & 11.54 \\
\hline Class I obese $\left(30.0 \mathrm{~kg} / \mathrm{m}^{2} \leq \mathrm{BMI}<35.0 \mathrm{~kg} / \mathrm{m}^{2}\right)$ & 70 & 26.92 \\
\hline Class II obese $\left(35.0 \mathrm{~kg} / \mathrm{m}^{2} \leq \mathrm{BMI}<40.0 \mathrm{~kg} / \mathrm{m}^{2}\right)$ & 57 & 21.92 \\
\hline Class III obese $\left(\mathrm{BMI} \geq 40.0 \mathrm{~kg} / \mathrm{m}^{2}\right)$ & 103 & 39.62 \\
\hline
\end{tabular}

GED, General Educational Development.

Percentages might not add up to $100 \%$ due to rounding or missing data.

The average BMI was 39.02 (sD 8.66$) \mathrm{kg} / \mathrm{m}^{2}$, ranging from $25 \cdot 08$ to $75 \cdot 35 \mathrm{~kg} / \mathrm{m}^{2}$.

\section{Daily fruit and vegetable intake}

Fruit and vegetable intake as measured using the BRFSS FV module was on average 2.79 (SD 1.88) servings/d, ranging from a minimum of 0.09 to a maximum of 9.32 servings/d (Table 2). The average intake from the recalls was slightly lower (mean $=2.66$ servings $/$ d) but had a higher standard deviation $(\mathrm{SD}=2.66$ servings $/ \mathrm{d})$ and a range from zero to 27.65 servings/d. Looking at fruit and vegetable intake by BMI, education and age, there were surprisingly small differences in average intake but large standard deviations across all subgroups.

The deattenuated correlation coefficient for fruit and vegetable intake for the overall sample was low $(r=0 \cdot 22)$. There was large variation across BMI categories, with the overweight category having the highest validity $(r=0.53)$. The deattenuated correlation coefficient for the class I and II obese participants $\left(30 \cdot 0 \mathrm{~kg} / \mathrm{m}^{2} \leq \mathrm{BMI}<40.0 \mathrm{~kg} / \mathrm{m}^{2}\right)$ and class III obese participants (BMI $\geq 40 \cdot 0 \mathrm{~kg} / \mathrm{m}^{2}$ ) were low $(r=0.19$ and $r=0.23$, respectively). When looking at education strata, the validity was extremely low for those participants with less than a high-school diploma $(r=0 \cdot 05)$ and highest for those with some college education $(r=0.45)$.
The age group with the lowest validity was older women aged 55-65 years $(r=0 \cdot 07)$. The deattenuated correlation coefficient was higher among younger women, with $r=0.32$ among those 35-45 years of age and $r=0.32$ among those $45-55$ years of age. The validity for unemployed participants $(r=0.25)$ was similar to that for employed participants $(r=0 \cdot 20)$. There were no clear patterns in deattenuated correlation coefficients observed across subgroups.

The Bland-Altman plot in Fig. 1 shows the mean difference between the two methods along with the $95 \%$ limits of agreement. For fruit and vegetable intake, the plot illustrates limited agreement between the instruments for some individuals at higher intake levels; i.e. for some participants with high mean of levels of fruit and vegetable intake as measured by the two instruments, the differences in fruit and vegetable intake measurements are quite large, some with the $24 \mathrm{~h}$ dietary recall values being much larger than the BRFSS and vice versa. The variability in measurement increases with larger mean fruit and vegetable intake.

\section{Percentage of daily energy from fat}

The mean percentage of energy from fat measured with the NCI fat screener was 33.04 (SD 4.27)\%, ranging from 
Table 2 Mean daily servings of fruits and vegetables from two $24 \mathrm{~h}$ dietary recalls and the BRFSS FV module, and corresponding deattenuated Pearson correlation coefficients between the two methods, among African-American women ( $n 260)$ aged 35-65 years, southwest Georgia, USA, February 2011-December 2012

\begin{tabular}{|c|c|c|c|c|c|c|c|c|}
\hline \multirow[b]{2}{*}{ Group } & \multirow[b]{2}{*}{$n$} & \multicolumn{2}{|c|}{$24 \mathrm{~h}$ recalls $†$} & \multicolumn{2}{|c|}{ BRFSS FV module } & \multicolumn{2}{|c|}{ Differenceł } & \multirow{2}{*}{$\begin{array}{l}\text { Deattenuated correlation } \\
\text { coefficient, } r\end{array}$} \\
\hline & & Mean & SD & Mean & SD & Mean & SD & \\
\hline All & 260 & 2.66 & $2 \cdot 66$ & 2.79 & 1.88 & 0.12 & 2.93 & $0.22^{\star \star \star}$ \\
\hline \multicolumn{9}{|l|}{ Weight category } \\
\hline Overweight & 30 & 2.06 & 1.53 & 2.90 & $2 \cdot 39$ & 0.83 & $2 \cdot 15$ & $0.53^{\star \star}$ \\
\hline Class I and II obese & 127 & $2 \cdot 87$ & 3.22 & 2.89 & 1.86 & 0.02 & 3.42 & $0.19^{\star}$ \\
\hline Class III obese & 103 & $2 \cdot 58$ & 2.09 & 2.63 & 1.74 & 0.05 & 2.42 & $0.23^{*}$ \\
\hline \multicolumn{9}{|l|}{ Education } \\
\hline Less than high-school diploma or GED & 56 & $2 \cdot 17$ & 1.93 & $2 \cdot 51$ & 1.85 & 0.33 & $2 \cdot 61$ & 0.05 \\
\hline High school or GED & 96 & 2.92 & 3.27 & 2.78 & 1.99 & -0.13 & 3.56 & $0 \cdot 16$ \\
\hline Some college/technical school & 76 & 2.54 & 2.55 & $2 \cdot 72$ & 1.73 & 0.18 & 2.50 & $0.45^{\star \star \star}$ \\
\hline College graduate & 31 & 3.08 & 1.84 & 3.51 & 1.84 & 0.43 & $2 \cdot 36$ & 0.18 \\
\hline \multicolumn{9}{|l|}{ Age (years) } \\
\hline $35-45$ & 80 & $2 \cdot 62$ & $2 \cdot 60$ & $2 \cdot 82$ & 1.99 & 0.20 & $2 \cdot 83$ & $0.32^{\star \star}$ \\
\hline $45-55$ & 101 & $2 \cdot 72$ & $2 \cdot 18$ & $2 \cdot 87$ & $2 \cdot 06$ & 0.15 & 2.54 & $0.32^{\star \star}$ \\
\hline $55-65$ & 79 & $2 \cdot 64$ & 3.26 & $2 \cdot 65$ & 1.51 & 0.01 & 3.49 & 0.07 \\
\hline \multicolumn{9}{|l|}{ Employment status } \\
\hline Not employed & 143 & 2.59 & $2 \cdot 00$ & $2 \cdot 78$ & 1.91 & 0.18 & 2.49 & $0.25^{\star \star}$ \\
\hline Employed & 117 & $2 \cdot 75$ & $3 \cdot 31$ & $2 \cdot 80$ & 1.85 & 0.05 & 3.41 & $0.20^{\star}$ \\
\hline
\end{tabular}

BFRSS FV module, Behavioral Risk Factor Surveillance System's Fruit and Vegetable Consumption Module; GED, General Educational Development. ${ }^{\star} P<0.05,{ }^{* \star} P<0.01,{ }^{* \star \star} P<0.001$.

†The average fruit and vegetable intake as assessed with the $24 \mathrm{~h}$ dietary recalls was slightly higher (2.82) when including French fries; the deattenuated correlation coefficient was slightly smaller $(0 \cdot 22)$.

fDifference was calculated by subtracting the $24 \mathrm{~h}$ recall intake from the BRFSS intake.

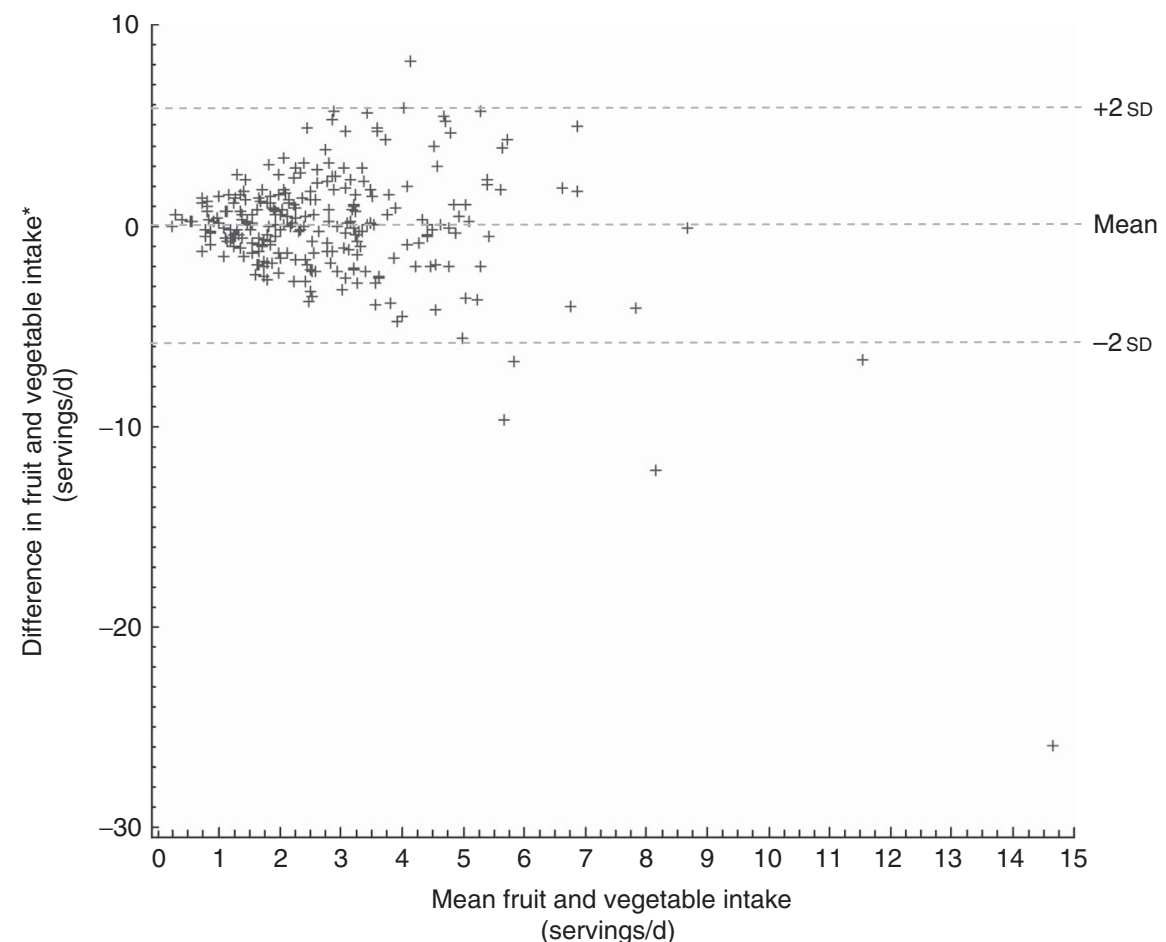

Fig. 1 Bland-Altman plot of fruit and vegetable intake as measured by $24 \mathrm{~h}$ dietary recalls and the BFRSS FV module, displaying the difference in fruit and vegetable intake depending on the mean of fruit and vegetable intake as measured by the two instruments for African-American women ( $n$ 260) aged 35-65 years, south-west Georgia, USA, February 2011-December 2012. *Note: difference was calculated by subtracting the $24 \mathrm{~h}$ recall intake from the BRFSS intake (BFRSS FV module, Behavioral Risk Factor Surveillance System's Fruit and Vegetable Consumption Module)

$16 \cdot 04 \%$ to $44.67 \%$ (Table 3 ). The $24 \mathrm{~h}$ dietary recall intake was on average higher by about $2.5 \%$. The standard deviation of the NDSR intake was much larger $(\mathrm{SD}=8.29 \%)$ and the range extended further both at the lower (14.29\%) and upper ends (61.19\%). Looking at education, age and employment status, the NCI fat screener underestimated 
Table 3 Mean percentage of energy from fat from two $24 \mathrm{~h}$ dietary recalls and the $\mathrm{NCl}$ fat screener, and corresponding deattenuated Pearson correlation coefficients between the two methods, among African-American women $(n 260)$ aged $35-65$ years, south-west Georgia, USA, February 2011-December 2012

\begin{tabular}{|c|c|c|c|c|c|c|c|c|}
\hline \multirow[b]{2}{*}{ Group } & \multirow[b]{2}{*}{$n$} & \multicolumn{2}{|c|}{$24 \mathrm{~h}$ recalls } & \multicolumn{2}{|c|}{$\mathrm{NCl}$ fat screener } & \multicolumn{2}{|c|}{ Difference† } & \multirow{2}{*}{$\begin{array}{c}\text { Deattenuated correlation } \\
\text { coefficient, } r\end{array}$} \\
\hline & & Mean & SD & Mean & SD & Mean & SD & \\
\hline All & 260 & 35.50 & 8.29 & 33.04 & $4 \cdot 27$ & $-2 \cdot 46$ & 7.82 & $0.38^{\star \star \star}$ \\
\hline \multicolumn{9}{|l|}{ Weight category } \\
\hline Overweight & 30 & 33.68 & 9.57 & 34.21 & 3.66 & 0.54 & 7.83 & $0.65^{\star * *}$ \\
\hline Class I and II obese & 127 & $35 \cdot 70$ & 8.08 & $32 \cdot 71$ & $4 \cdot 15$ & -2.99 & 7.90 & $0.31^{* * *}$ \\
\hline Class III obese & 103 & 35.79 & $8 \cdot 16$ & 33.12 & 4.55 & -2.67 & 7.60 & $0.43^{\star \star \star}$ \\
\hline \multicolumn{9}{|l|}{ Education } \\
\hline Less than high-school diploma or GED & 56 & 36.55 & $7 \cdot 78$ & 33.24 & 4.52 & -3.30 & 7.01 & $0.47^{\star * *}$ \\
\hline High school or GED & 96 & $35 \cdot 20$ & 9.03 & 32.60 & 4.48 & -2.60 & $8 \cdot 18$ & $0.45^{\star \star *}$ \\
\hline Some college/technical school & 76 & $35 \cdot 32$ & 8.29 & 33.37 & 3.95 & -1.95 & $8 \cdot 28$ & $0.25^{\star}$ \\
\hline College graduate & 31 & 35.05 & 7.01 & 33.30 & 4.02 & -1.75 & $7 \cdot 22$ & 0.24 \\
\hline \multicolumn{9}{|l|}{ Age (years) } \\
\hline $35-45$ & 80 & $35 \cdot 21$ & 8.68 & 33.37 & 4.52 & -1.84 & 7.56 & $0.52^{* * *}$ \\
\hline $45-55$ & 101 & 35.97 & 8.84 & 33.12 & 4.43 & -2.84 & 8.76 & $0.28^{\star *}$ \\
\hline $55-65$ & 79 & $35 \cdot 20$ & $7 \cdot 15$ & $32 \cdot 61$ & 3.79 & -2.59 & 6.81 & $0.36^{\star *}$ \\
\hline \multicolumn{9}{|l|}{ Employment status } \\
\hline Not employed & 143 & $35 \cdot 36$ & 8.37 & $32 \cdot 79$ & 4.68 & -2.58 & $8 \cdot 10$ & $0.44^{\star \star \star}$ \\
\hline Employed & 117 & 35.67 & 8.22 & 33.36 & 3.70 & $-2 \cdot 31$ & 7.50 & $0.34^{\star \star \star}$ \\
\hline
\end{tabular}

$\mathrm{NCl}$ fat screener, National Cancer Institute's Percentage Energy from Fat Screener; GED, General Educational Development. ${ }^{\star} P<0.05,{ }^{* \star} P<0.01,{ }^{\star \star \star} P<0.001$.

†Difference was calculated by subtracting the $24 \mathrm{~h}$ recall percentage from the $\mathrm{NCl}$ percentage.

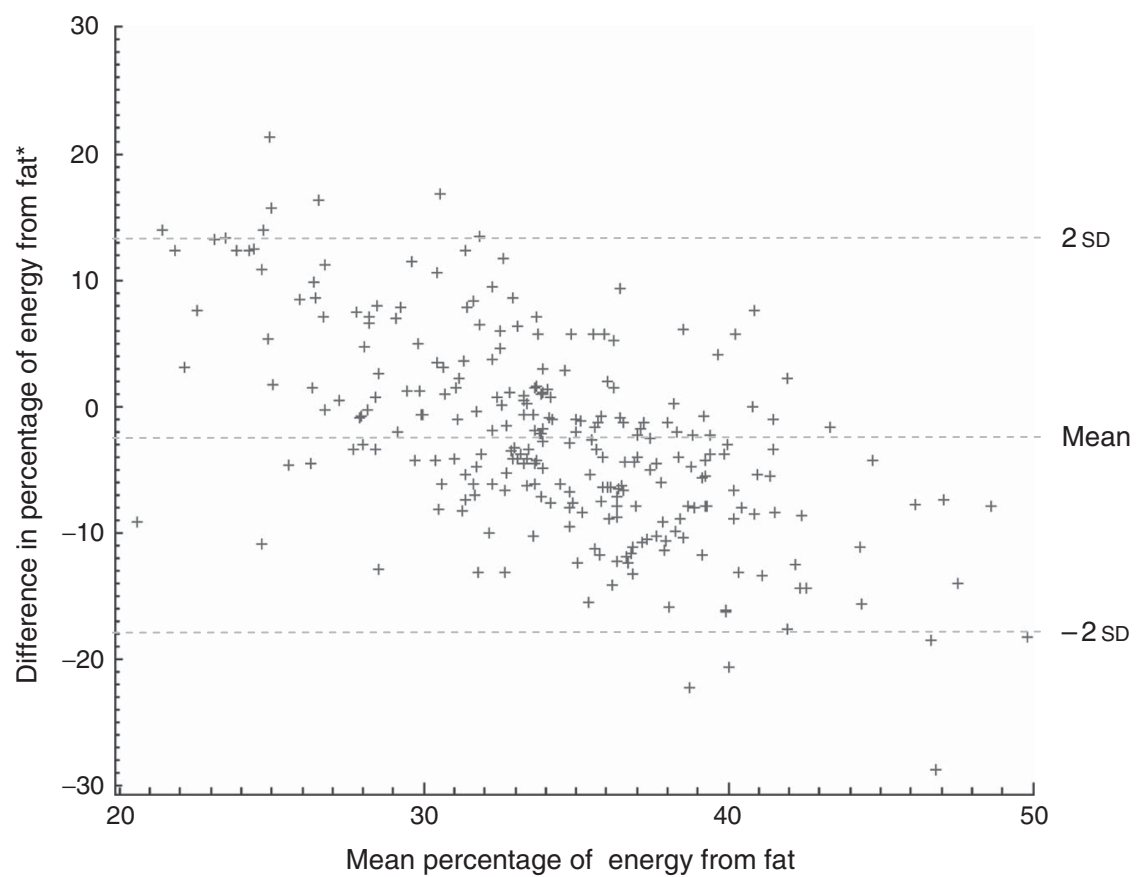

Fig. 2 Bland-Altman plot of fat intake as measured by $24 \mathrm{~h}$ dietary recalls and the $\mathrm{NCl}$ fat screener, displaying the difference in fat intake depending on the mean fat intake as measured by the two instruments for African-American women ( $n$ 260) aged 35-65 years, south-west Georgia, USA, February 2011-December 2012. *Note: difference was calculated by subtracting the $24 \mathrm{~h}$ recall percentage from the $\mathrm{NCl}$ percentage ( $\mathrm{NCl}$ fat screener, National Cancer Institute's Percentage Energy from Fat Screener)

intake compared with the $24 \mathrm{~h}$ recalls in almost all subgroups: from $1.75 \%$ (college graduates) to $3 \cdot 30 \%$ (those with less than a high-school diploma). The only exceptions were overweight women for whom the NCI fat screener overestimated fat intake by about $0.5 \%$. The standard deviation of fat intake measured with the NCI fat screener was moderately high in all subgroups, ranging from $3.66 \%$ to $4.68 \%$.

The deattenuated correlation coefficient for the percentage of daily energy from fat for the overall sample was moderate at $r=0 \cdot 38$. Validity was highest among overweight participants $(r=0.65)$ and lowest among obese 
class I and II $\left(30 \cdot 0 \mathrm{~kg} / \mathrm{m}^{2} \leq \mathrm{BMI}<40 \cdot 0 \mathrm{~kg} / \mathrm{m}^{2}\right)$ study participants $(r=0 \cdot 31)$. However, the deattenuated correlation coefficient among participants in the class III obesity category (BMI $\left.\geq 40.0 \mathrm{~kg} / \mathrm{m}^{2}\right)$ was higher $(r=0.43)$ compared with that in obese class I and II. Validity decreased with an increase in education from $r=0.47$ for women with less than a high-school diploma to $r=0.24$ for college graduates. Validity was highest for the youngest group of participants, aged 35-44 years $(r=0.52)$, and lowest for the middle group, aged $45-54$ years $(r=0 \cdot 28)$. Similar to the fruit and vegetable intake, there were no clear patterns in deattenuated correlation coefficients for percentage of daily energy from fat in all subgroups.

The Bland-Altman plot in Fig. 2 shows the mean difference between the two methods along with the $95 \%$ limits of agreement. There did not appear to be any consistent bias across levels of fat intake; i.e. for participants with low, medium or high levels of fat intake (mean between $24 \mathrm{~h}$ recall and $\mathrm{NCI}$ fat screener), the difference in measurement does not seem to vary systematically. However, the plot suggests a trend. With higher mean percentage of energy from fat, the difference between the NCI percentage and the $24 \mathrm{~h}$ recall percentage becomes more negative. Also, more positive outliers in differences in scores were present for low mean intake percentages and more negative outliers for high mean intake percentages.

\section{Discussion}

Our study of low-income overweight and obese AfricanAmerican women provides valuable information about the validity of two commonly used measures to assess dietary intake. We observed relatively low overall correlations between fruit and vegetable intake measured by the BRFSS FV module and the repeated $24 \mathrm{~h}$ recalls. The BRFSS FV module does not include portion size information which may be one reason for the observed differences, particularly at the higher intake levels. It may be that respondents report instances of even small amounts of fruits and vegetables in the BRFSS measure and due to the lack of adjustment for portion size, actual consumption is over-reported. Our results showing that the FV module overestimated fruit and vegetable intake are consistent with previous findings including those of the original BRFSS validation study ${ }^{(26,27)}$. It is also possible that when responding to the BRFSS FV module respondents overestimated fruits and vegetables consumed as part of mixed dishes. The NDSR $24 \mathrm{~h}$ dietary recall does include actual amounts of fruits and vegetables from mixed dishes in calculating total fruit and vegetable servings, while the BRFSS estimates each instance as one portion.

In terms of determining the percentage of daily energy from fat, we observed that the NDSR $24 \mathrm{~h}$ dietary recall was higher than the NCI fat screener. Furthermore, the underestimation of the NCI fat screener varied by age, education and employment status. There may be a number of potential explanations for the discrepancy between fat intakes as estimated by the two methods. The NCI fat screener was developed using data from the nationally representative US 1989-91 Continuing Survey of Food Intakes by Individuals ${ }^{(10)}$. The instrument includes the strongest food group predictors of percentage energy from fat based on this sample. The development and the finalized instrument consider frequency of consumption but not portion sizes for the foods/food groups. It is well known that food portion sizes have increased over time. Our population may have been consuming larger portion sizes of higher-fat items than the original reference population, resulting in an underestimate of fat intake. In addition, the NCI instrument may not capture some common uses of added fat for southern US-based populations, such as animal (meat) fat used to sauté vegetables or season cooked greens, fat added to cooked grits and gravies added to biscuits and cooked meats. Some high-fat foods such as barbequed meats (e.g. ribs) and fried meats and poultry may be eaten more frequently in our population than others and are not included in the NCI fat screener. Lastly, the NDSR specifically asks about fat additions to individual foods such as breads and vegetables during the interview as these items are reported. This may result in a more complete estimate of fats added to foods with NDSR as compared with the NCI fat screener.

Other studies have compared the screening instrument for fat intake used in the present study with repeated $24 \mathrm{~h}$ recalls in a variety of different settings and populations; however, the sociodemographic and health characteristics of our rural southern US population are unique. In the original validation paper for the fat screener, Thompson et al. compared results from the screener with data from two telephone $24 \mathrm{~h}$ dietary recalls ${ }^{(10,11)}$. A sub-sample of 404 participants of both genders from the National Institutes of Health-AARP (American Association of Retired Persons) Diet and Health Study completed the screener, $24 \mathrm{~h}$ dietary recalls and provided demographic data. Compared with our study, the participants in that study were older (50-69 years) and more likely to be Caucasian and of higher socio-economic status. Similar to what we observed, the screener tended to estimate lower fat intakes than the $24 \mathrm{~h}$ recalls. Among women, the recalls estimated mean percentage of energy from fat at $31.3 \%$ and the screener at $28.4 \%$. After applying a measurement error model the investigators reported correlations between the two methods of 0.64 and 0.58 for men and women, respectively. This is much higher than the correlations we observed (0.38).

In the original validation study of the BRFSS FV module by Serdula et al., the investigators compared it with $7 \mathrm{~d}$ food records in an Arizona population of fifty-one adults $(r=0.34)$ and with results from a series of four $2 \mathrm{~d}$ diet records (eight in total; $r=0.66$ ) collected quarterly in a 
Wisconsin population of 169 adults $^{(9)}$. Overall their populations reported higher fruit and vegetable consumption (means of 3.9 and 4.2 daily servings) with the BRFSS module than we observed in the present study (mean 2.79). Interestingly, for the Wisconsin group, diet records found higher intakes of fruit and vegetables (mean $4 \cdot 3)$ and for the Arizona group, diet records found lower intakes of fruits and vegetables (mean 2.4) than the respective BRFSS values. The additional days of dietary data may in part explain the more robust correlations observed in these study populations compared with our own results $(r=0.22)$. To our knowledge there are no other published validation studies for this instrument. Investigators have, however, compared the longer NCI fruit and vegetable screener (sixteen-, seventeen- and nineteen-item versions $v$. six items for BRFSS) with dietary recall data and the majority of results were consistent with those presented here (i.e. screeners consistently underreporting fruit and vegetable consumption) ${ }^{(10,27,28)}$.

Our study has at least three strengths. First, we compared $24 \mathrm{~h}$ recall data collected with the NDSR computer-directed dietary assessment and analysis program, the premiere software program for nutrition research, with data from two widely used nutrition screeners. Second, all of the interview staff involved in the research were trained to use NDSR and observed strict quality assurance procedures throughout the data collection process. Finally, trained telephone interviewers also collected the dietary screener data, thereby minimizing the possibility for respondents to misinterpret the items or skip questions. Despite the strengths of our study, a few limitations should be noted. First, all of the nutrition data were self-reported by our participants and could be subject to recall and social desirability bias. However, many of the responses confirmed low intake of fruits and vegetables overall. Low intakes of fruits and vegetables among our participants might impact the validity of the BRFSS FV module for this and similar populations because of the difficulty in reporting usual intake of foods consumed infrequently. Furthermore, the narrow range of fruit and vegetable intake could also have resulted in the relatively weak correlations. Our reference standard, the $24 \mathrm{~h}$ dietary recall, is not without limitations. For example, two recalls may not be sufficient to accurately estimate usual intake for some nutrients and particularly for episodically consumed foods ${ }^{(5,7,29)}$. It is also possible that the $24 \mathrm{~h}$ recalls may be more prone to reporting biases among our study participants because of their weight status or educational attainment. We advise other researchers to give careful consideration before judging that a screener is an appropriate measure for both fruit and vegetable intake and fat intake in intervention studies of modest size. In addition, the modest sample size, especially in some of the subgroups, is a limitation.

Future research using screeners to measure fruit and vegetable consumption should attempt to quantify fruits and vegetables included in mixed dishes. Screeners that target fat intake may want to include instructions to remind research participants to include common sources of added fat intake when responding to items. Alternatively, future research could consider utilizing or modifying instruments or processes for determining fat intake obtained from vegetables, soups and gravies seasoned with fatty meat products. These fats are widely used among those in rural areas and those who are low income. Studies would need to be conducted to determine a modified or new instrument's validity. Lastly, future studies should attempt to examine how well screening instruments capture intervention-related dietary changes or other fluctuations that occur in dietary patterns over time.

\section{Acknowledgements}

Acknowledgements: The authors wish to thank members of the Emory Prevention Research Center's Community Advisory Board for their guidance in the design and implementation of this research. They also wish to thank the interviewers, study participants and community health centre partners for their valuable contributions to this research. Financial support: This publication was supported by Cooperative Agreement \#5U48DP001909 from the Centers for Disease Control and Prevention. The findings and conclusions in this article are those of the authors and do not necessarily represent the official position of the Centers for Disease Control and Prevention. Conflict of interest: None. Authorship: I.A., R.H., J.A.G., B.G. and M.C.K. conceptualized the larger study. I.A., R.H. and T.J.H. formulated the specific research questions for the present study. I.A. managed data collection. R.H. performed the analysis. All authors wrote sections of the paper and interpreted the results. All authors reviewed and edited the full manuscript. Ethics of human subject participation: This study was reviewed and approved by the Emory University Institutional Review Board.

\section{References}

1. Flegal KM, Carroll MD, Kit BK et al. (2012) Prevalence of obesity and trends in the distribution of body mass index among US adults, 1999-2010. JAMA 307, 491-497.

2. Jackson JE, Doescher MP, Jerant AF et al. (2005) A national study of obesity prevalence and trends by type of rural county. J Rural Health 21, 140-148.

3. Befort CA, Nazir N \& Perri MG (2012) Prevalence of obesity among adults from rural and urban areas of the United States: findings from NHANES (2005-2008). J Rural Health 28, 392-397.

4. Perez-Escamilla R, Obbagy JE, Altman JM et al. (2012) Dietary energy density and body weight in adults and children: a systematic review. J Acad Nutr Diet 112, 671-684.

5. Resnicow K, Odom E, Wang T et al. (2000) Validation of three food frequency questionnaires and 24-hour recalls with serum carotenoid levels in a sample of AfricanAmerican adults. Am J Epidemiol 152, 1072-1080. 
6. Mitchell DC, Tucker KL, Maras J et al. (2012) Relative validity of the Geisinger Rural Aging Study food frequency questionnaire. J Nutr Health Aging 16, 667-672.

7. Cade J, Thompson R, Burley V et al. (2002) Development, validation and utilisation of food-frequency questionnaires a review. Public Health Nutr 5, 567-587.

8. Buzzard M (1998) 24-hour dietary recall and food record methods. In Nutritional Epidemiology, pp. 50-73 [WC Willett, editor]. New York: Oxford University Press.

9. Serdula M, Coates R, Byers T et al. (1993) Evaluation of a brief telephone questionnaire to estimate fruit and vegetable consumption in diverse study populations. Epidemiology $\mathbf{4}$, 455-463.

10. Thompson FE, Midthune D, Subar AF et al. (2007) Development and evaluation of a short instrument to estimate usual dietary intake of percentage energy from fat. J Am Diet Assoc 107, 760-767.

11. Thompson FE, Midthune D, Williams GC et al. (2008) Evaluation of a short dietary assessment instrument for percentage energy from fat in an intervention study. $J$ Nutr 138, issue 1, 193S-199S.

12. Field AE, Colditz GA, Fox MK et al. (1998) Comparison of 4 questionnaires for assessment of fruit and vegetable intake. Am J Public Health 88, 1216-1218.

13. Smith-Warner SA, Elmer PJ, Fosdick L et al. (1997) Reliability and comparability of three dietary assessment methods for estimating fruit and vegetable intakes. Epidemiology 8, 196-201.

14. Williams GC, Hurley TG, Thompson FE et al. (2008) Performance of a short percentage energy from fat tool in measuring change in dietary intervention studies. J Nutr 138, issue $1,212 S-217 S$.

15. Snyder DC, Sloane R, Lobach D et al. (2004) Agreement between a brief mailed screener and an in-depth telephone survey: observations from the Fresh Start study. J Am Diet Assoc 104, 1593-1596.

16. Marks GC, Hughes MC \& van der Pols JC (2006) The effect of personal characteristics on the validity of nutrient intake estimates using a food-frequency questionnaire. Public Health Nutr 9, 394-402.

17. Marks GC, Hughes MC \& van der Pols JC (2006) Relative validity of food intake estimates using a food frequency questionnaire is associated with sex, age, and other personal characteristics. J Nutr 136, 459-465.
18. Block G \& Hartman AM (1989) Issues in reproducibility and validity of dietary studies. Am J Clin Nutr 50, 1133-1138.

19. Centers for Disease Control and Prevention (2005) Behavioral Risk Factor Surveillance System Survey Questionnaire [US Department of Health and Human Services, editor]. Atlanta, GA: CDC.

20. Schakel SF, Sievert YA \& Buzzard IM (1988) Sources of data for developing and maintaining a nutrient database. $\mathrm{J} \mathrm{Am}$ Diet Assoc 88, 1268-1271.

21. Schakel SF, Buzzard IM \& Gebhardt SE (1997) Procedures for estimating nutrient values for food composition databases. J Food Compost Anal 10, 102-114.

22. Schakel SF (2001) Maintaining a nutrient database in a changing marketplace: keeping pace with changing food products - a research perspective. J Food Compost Anal 14, 315-322.

23. Kegler MC, Escoffery C, Alcantara I et al. (2008) A qualitative examination of home and neighborhood environments for obesity prevention in rural adults. Int J Behav Nutr Phys Act 5, 65.

24. Beaton GH, Milner J, McGuire V et al. (1983) Source of variance in 24-hour dietary recall data: implications for nutrition study design and interpretation. Carbohydrate sources, vitamins, and minerals. Am J Clin Nutr 37, 986-995.

25. Bland JM \& Altman DG (1986) Statistical methods for assessing agreement between two methods of clinical measurement. Lancet 1, 307-310.

26. Kim DJ \& Holowaty EF (2003) Brief validated survey instruments for the measurement of fruit and vegetable intakes in adults: a review. Prev Med 36, 440-447.

27. Yaroch AL, Tooze J, Thomson FE et al. (2012) Evaluation of three short dietary instruments to assess fruit and vegetable intake: the National Cancer Institute's Food Attitudes and Behaviors Survey. J Acad Nutr Diet 112, $1570-1577$.

28. George SM, Thompson FE, Midthune D et al. (2012) Strength of the relationships between three self-reported dietary intake instruments and serum carotenoids: the Observing Energy and Protein Nutrition (OPEN) Study. Public Health Nutr 15, 1000-1007.

29. Ma Y, Olendzki BC, Pagoto SL et al. (2009) Number of 24-hour diet recalls needed to estimate energy intake. Ann Epidemiol 19, 553-559. 